\title{
On Susan Schneider's Artificial You: the twin problem of AI and lab-grown brain consciousness
}

\author{
Ho Manh Tung \\ Ritsumeikan Asia Pacific University \\ Beppu, Oita, Japan
}

November 4, 2020

A recent article in Nature discusses the problem of consciousness in lab-grown cerebral organoids (Reardon, 2020). In the case of lab-grown brains, it seems very intuitively likely that they will become conscious once certain level of mass is achieved. In the case of artificial intelligence (AI), given their non-biological nature, it is harder to imagine how AI can become conscious. There is no better place to start educating yourself about the problem of AI consciousness than Susan Schneider's Artificial You: AI and the future of your mind (2019).

The book starts with classifying two mainstream positions regarding AI consciousness: biological naturalism and techno-optimism. Biological naturalism views consciousness is an intrinsic property of biological systems and it is impossible to replicate it in non-biological systems like machines or computers, roughly speaking. Techno-optimism is the view held by many contemporary AI experts, who think that given that most, if not all, processes in the brain are computational, our technological and scientific advances will eventually deliver AI consciousness. Then Schneider proposes the middle way: The wait-and-see approach, in which, she recognizes the validity of the arguments of both sides, yet, whether we will have conscious AI will depend on so many unknown unknowns: political changes, PR disasters, and most 
important of all, scientific discoveries. That means being totally committed to one side is a mistake.

Schneider is right to point out the computational theory of the brain is ultimately research paradigm that is prominent in cognitive science, an interdisciplinary science of the mind and the brain. One should not confuse that theory and think that all there are to the brain is computational. I agree that having the true understanding of what constitutes the mind, i.e., the metaphysical nature of the mind, matters enormously for our future of living with intelligent machines and becoming brain-enhanced ourselves. For example, if consciousness is purely about certain patterns of information processing, i.e., the viewpoint called "patternism," then there is a possibility that we could upload our minds into the cloud. Or if AI can become conscious, robot right is a serious ethical issue.

Schneider also provides an assessment of the view that "the mind is a software that the brain runs," which is a popular view among the techno-optimists and even among laypeople. This so-called the Software view suggests the mind is a software program, a long string of codes of sort, hence, an abstract entity. But in philosophy of mathematics, abstract entities are said to be nonconcrete: nonspatial, nontemporal, nonphysical, and acausal. Because, entities that have a mind are spatial and causal beings, it indicates the Software view is a categorical mistake. This is the reason Schneider rejects the Software view.

Schneider does offer an adjustment to the Software view: "the mind is the entity running the program (where a program is an algorithm that the brain and other cognitive system implements, something in principle discoverable by cognitive science." This is the Software Instantiation Approach to the Mind (SIM*). This approach avoids the categorical mistake of the 
Software view, but, it still does not say anything of substance about the nature of the mind, other the mind is separate from the software of the brain.

Indeed, the fundamental mystery of consciousness will be in more pressing needs of solution due to the advances in AI and how AI systems become more pervasive in our daily life. The problem is understanding how consciousness arises in human brains tells us nothing about how it can arise in non-biological system. Even if we have a complete map of neural correlation of conscious experiences (Michel et al., 2019), which is still far away into the future, this map does not readily translate into the silicon-based wiring of AI.

Here, I think the literature on machine consciousness can benefit from differentiating three accounts of consciousness: the physical, biological, and computational. It seems to me a biological creature, given it must compete for foods, shelters, and mating opportunities, can benefit from greater and greater consciousness of the external environment and itself. Thus, it is fairly straightforward that animals need to evolve the ability of awareness.

Even the evolutionary biological account of consciousness seems clear, one wonders how it works for the computational and physical accounts. When considering things from a computational perspective, it seems no consciousness is required at all as all problems can be solved algorithmically. However, one can also argue, all problems can be solved algorithmically only when knowledge required to solve such problems has already been created and instantiated. Knowledge can be instantiated in a piece of linguistic thought of a human, in a strand of a DNA genetic code, or in a machine. This line of reasoning leaves us with whether creative problemsolving require conscious awareness. 
The issue of artificial creativity is discussed extensively in the chapter of the same name in David Deustch's The beginning of infinity (2011, pp.148-163). Here, Deutsch argues the field of AI has made no progress because it has not solved its central philosophical problem: how creativity works. Indeed, the ability to take a step back, to reflect on multiple possible paths to one's solution, or to imagine an analogous easier problem (Polya, 2015), or to recognize how a serendipitous idea can reframe the problem (Vuong \& Napier, 2014), etc., seems to require conscious awareness.

Indeed, it appears having consciousness can speed up the creative problem-solving process. To the extent that creativity helps with survival and fitness, evolution of computational programs might bring consciousness along. In other words, without a prerequisite evolutionary setting being put in place, it seems hard to conceive of a computational system bootstrapping itself into being self-aware.

It seems that the biological and computational account share two prerequisites: an evolutionary dynamic and certain level of complexity. With the maturing of complexity science, we can anticipate certain problems of consciousness will be clarified and explained in the future (Arsiwalla \& Verschure, 2018). The hardest problem is the physical account of consciousness, where there seems to be no way of explaining how inanimate matters become conscious or whether the matters might already have minds as most of them cannot communicate.

In conclusion, with the advances in computer science and biological engineering, the twin problems of AI consciousness and lab-grown brain consciousness are here to stay. These problems challenge human scientific ingenuity to the utmost limit. To avoid creating untold suffering for these entities, ethical guidelines in these engineering and scientific endeavors are much needed (Reardon, 2020). It seems to me simple utilitarian calculation would be a mistake, 
as so much is unknown about the problem of consciousness. Here, the principles of open science and intellectual humility must be upheld to the highest standard (Vuong et al., 2018; Vuong, 2020)

\section{References}

Arsiwalla, X. D., \& Verschure, P. (2018). Measuring the Complexity of Consciousness. Frontiers in Neuroscience, 12(424). doi:10.3389/fnins.2018.00424

Deutsch, D. (2011). The beginning of infinity: Explanations that transform the world. London, UK: Penguin.

Michel, M., Beck, D., Block, N., Blumenfeld, H., Brown, R., Carmel, D., ... \& Dehaene, S. (2019). Opportunities and challenges for a maturing science of consciousness. Nature Human Behaviour, 3(2), 104-107.

Polya, G. (2015). How to Solve It: A New Aspect of Mathematical Method (Princeton Science Li edition ed.). Princeton: Princeton University Press.

Reardon, S. (2020). Can lab-grown brains become conscious? Nature, 568(658-661). doi:https://doi.org/10.1038/d41586-020-02986-y

Schneider, S. (2020). Artificial You: AI and the future of your mind. Princeton: Princeton University Press.

Vuong, Q. H. (2020). Reform retractions to make them more transparent. Nature, 582(149). doi:https://doi.org/10.1038/d41586-020-01694-X 
Vuong, Q.-H., La, V.-P., Vuong, T.-T., Ho, M.-T., Nguyen, H.-K. T., Nguyen, V.-H., . . . Ho, M.-T. (2018). An open database of productivity in Vietnam's social sciences and humanities for public use. Scientific Data, 5(1), 180188. doi:10.1038/sdata.2018.188 